Methods One hundred and sixty six children with Down Syndrome less than 16 years were collected from regional hospitals within one year (January to December 2020). We took history, reviewed medical records and performed clinical examination. We analyzed demographic characteristics, attitude and healthcare knowledge of caregivers, screening tests and following up conditions including problems in coping of caring the children with Down Syndrome.

Results Results: Of the 166 participants, majority, (53\%) were male. A total of 95(57.2\%) were from urban area and most of PDS (96.4\%) were cared by biological parents. Half of caregivers in this study group were nongraduate (most are middle-school education). Although (80.7\%) were born at regional hospitals, some were unaware of Down Syndrome until the child developed clinical symptoms (maximum age up to 9 years) and (45\%) have lack of knowledge about associated health problems. The most common medical conditions reported were cardiac (53.01\%), thyroid (13.25\%), visual (13.25\%), hearing problems (8.43\%).Screening tests done for cardiac, thyroid, vision and hearing were $72.89 \%, 40.96 \%$, $25.3 \%$ and $26.51 \%$ respectively and only $(21.38 \%)$ have regular follow up after diagnosis. Most of the caregivers have lack of knowledge about how to care their children, importance of screening tests according to age and regular follow up. Conditions such as financial difficulties, social problems and uneasily accessible health specialties especially for those who live in rural areas became barriers to receive proper health care services.

Conclusions More data are needed to understand health conditions, caregiver knowledge and explore the barriers in seeking proper health care services. Appropriate policies, feasible local guidelines and more education programmes to address health care challenges for Down Syndrome children are crucial to raise awareness of healthcare personals and reduce the gap to receive proper healthcare services. Screening tests according to age and long-term care programs are vastly underdeveloped in poor countries like Myanmar, it will be essential for complementing the support system and sustaining the major role that families or caregivers currently play.

\section{THE RELATIONSHIP OF LINGUAL FRENULUM AND OBSTRUCTIVE SLEEP APNOEA (OSA) IN CHILDREN}

Hoi Man Yuen, Chun Ting Au, Albert Martin Li, Kate Ching Ching Chan. Hong Kong

\subsection{6/bmjpo-2021-RCPCH.9}

Background Childhood obstructive sleep apnoea (OSA) is a prevalent disease, and reported to affect around 5\% of primary school-aged children. It is also clinically important as it can lead to neurocognitive, metabolic and cardiovascular complications. The tongue plays an important role in maintaining patency of the oropharynx. Lingual frenulum, a connective tissue between the floor of the mouth and the underside of the tongue, can affect the tongue position, its elevation and movements. Short lingual frenulum has been reported to be a risk factor for OSA. How frenulum length and its mobility affects craniofacial development and morphology remains to be defined.

Objectives In this study we aimed to prospectively evaluate the lingual frenulum length by free tongue measurement and tongue mobility in children suspected to have OSA. We hypothesized that OSA children would have shorter lingual frenulum than their non-OSA counterparts. Moreover, we explored the relationship between frenulum length and craniofacial profile using cephalometry. We hypothesized that cephalometric measurements would be different in children with and without short frenulum.

Methods Prepubertal Chinese children aged 5-12 years old, suspected to have OSA were recruited. Anthropometric measurements including weight, height, and circumferences of waist, hip and neck were taken on the day of admission. The lingual frenulum was evaluated based on tongue mobility and free tongue length. Tongue mobility obtained by a digital calliper was defined by Mpal/Mmax, which are the maximal distances between incisors during full mouth opening (Mmax) and when the tongue tip touched the palatal papilla (Mpal). The free tongue length was measured from the insertion of the lingual frenulum to the tongue tip using Quick Tongue-tie Assessment Tool (QTT). Normal tongue mobility was defined as mobility $\geq 50 \%$, and normal free tongue length was defined as $\geq 16 \mathrm{~mm}$. Cephalometric analysis was performed to evaluate the craniofacial profile. OSA was defined as obstructive apnoea hypopnoea index (OAHI) $\geq 1 / \mathrm{h}$ from overnight polysomnography.

Results In this study, 86 subjects (mean age: $8.36 \pm 1.69$ years) were recruited, and 50 were diagnosed to have OSA $(\mathrm{OAHI} \geq 1 / \mathrm{h})$. There was no significant difference in anthropometric measurements between OSA and non-OSA groups. The medians of the free tongue length in OSA and non-OSA groups were 20 and $24 \mathrm{~mm}(\mathrm{p}=0.321)$, respectively. The mean tongue mobility was $0.583( \pm 0.189)$ in OSA group, and $0.680( \pm 0.152)$ in non-OSA group $(p=0.010)$. Free tongue length was significantly correlated with most of the anthropometric variables including age, weight, height, BMI, waist, and hip circumferences, but did not correlate with any of the PSG variables. Tongue mobility was not correlated with any anthropometric variables, but inversely correlated with OAHI $(\mathrm{r}=-0.234, \mathrm{p}=0.030)$. In multivariate logistic regression, tongue mobility was independently associated with the presence of OSA after adjusted for age and gender. Tongue mobility was correlated with the cranial base angle $(\mathrm{Ba}-\mathrm{S}-\mathrm{N})$, which can affect the relative position of the mandible.

Conclusions Reduced tongue mobility is associated with OSA in prepubertal children. Furthermore, tongue mobility may be an important factor in driving mandibular development.

\section{ARE WE IN CONTROL OF OUR DEMONS? UNDERSTANDING COMPASSION SATISFACTION, COMPASSION FATIGUE AND BURNOUT IN AN ASIAN PAEDIATRIC EMERGENCY IN A PANDEMIC}

Kar Yee Catrin Kong, Sashikumar Ganapathy. Singapore

\subsection{6/bmjpo-2021-RCPCH.10}

Background The medical profession, with its remarkable physical and emotional demands, predisposes physicians to compassion fatigue (CF) and burnout (BO). Though these conditions have been studied individually, little attention has been paid to how pediatric emergency physicians experience these conditions in the context of an Asian emergency setting especially during a global pandemic.

Objectives In our study, we aim to understand the experiences of individual physicians and describe the potential triggers or 
protective factors of $\mathrm{CS}, \mathrm{BO}$ and compassion satisfaction (CS) amongst physicians in an Asian pediatric emergency department during a pandemic.

Methods A qualitative, individual interview methodology was employed. From March to April 2020, we enrolled 20 physicians involved in frontline care during the coronavirus disease 2019 (COVID-19) pandemic in the pediatric emergency department to participate. Semi-structured interviews were conducted, audiotaped and transcribed in verbatim, with identifiers removed. Themes were identified and data analysed using qualitative data analysis and iterative data analysis.

Results We recruited 20 physicians. Three themes emerged from data analysis. Firstly, we showed how workplace conditions are protective, including work morale, leadership and management and social support received. Secondly, workload affected participants emotionally and in terms of its heavier quantity and longer hours. Finally, intrinsic factors that were protective in developing $\mathrm{CF}$ or $\mathrm{BO}$ include having professional autonomy, experience, work-life balance and having emotional resilience to develop self-care. Cultural influences affect emotional regulation and can lead to negative coping with negative peer pressure. In general, participants quantified their levels of satisfaction at work as average to above-average. However, they highlighted experiencing greater stress during COVID-19 with the underlying fear of contagion and infection.

Conclusions Being a pediatric emergency physician puts one at greater risk of experiencing $\mathrm{CF}$ and $\mathrm{BO}$ because of work and non-work stressors especially during a global pandemic, influenced by sociocultural factors. A positive and supportive work environment should be created while providing culturallyadapted strategies to improve individual physician resilience to maintain their well-being

\section{CHALLENGES OF MONITORING AND TREATING RECURRENCE OF PRIMARY FOCAL SEGMENTAL GLOMERULOSCLEROSIS AFTER RENAL TRANSPLANTATION}

Christina Tran, Hilary Cramp. UK

\subsection{6/bmjpo-2021-RCPCH.11}

\section{Background}

Introduction In patients under 16 presenting with nephrotic syndrome, primary focal segmental glomerulosclerosis (FSGS) is an important differential. High recurrence rates following transplantation, and a considerable risk of progression to endstage renal disease, are well-recognised in cases with presumed circulating permeability factors rather than a genetic cause. To limit the risk of graft loss, it is therefore crucial to monitor for relapsing disease. The main marker of recurrence is proteinuria, however this can be challenging to interpret in a patient with high proteinuria pre-transplantation.

Objectives This is a case of a 14-year-old with FSGS, who developed early recurrence post-transplantation.

Methods

Case Presentation Pre-emptive renal transplantation was discussed with the family of a 14-year-old male with steroidresistant FSGS, who had also not responded to tacrolimus, mycophenolate and rituximab. Proteinuria would be difficult to interpret post-transplantation, as he continued to produce high levels of proteinuria with a significant urine output. A number of options were explored including; (1) bilateral nephrectomies or embolization pre-transplantation to shut down native function altogether; (2) a tube into the transplant renal pelvis passing through the bladder, serving as a catheter to monitor urine exclusively from the transplant kidney; (3) delay of transplantation until native kidney function completely ceased. Given the challenging practicality of (2), and uncertain timescale of (3), bilateral nephrectomies were arranged a few weeks prior to transplantation.

Results

Outcome After day five following a live donor kidney transplant, proteinuria developed progressively. Daily therapeutic plasma exchange was commenced for two weeks. Renal function continued to deteriorate, and lipoprotein apheresis was trialled. A transplant renal biopsy, nine days following lipoprotein apheresis, reported early recurrence of FSGS.

Conclusions

Discussion The leading cause of renal graft failure in primary FSGS, within the paediatric cohort, is disease recurrence. Removal of circulating permeability factors using extracorporeal systems, such as therapeutic plasma exchange and lipoprotein apheresis, could theoretically improve graft survival. Despite a number of possibilities considered, this case highlights the difficulties of monitoring and treating disease recurrence post-transplantation.

\section{DEVELOPMENT OF PERIOPERATIVE CHECKLIST FOR INFANTS REQUIRING SURGICAL INTERVENTION}

Vaibhav Katiyar, Theodore Dassios, Ravindra Bhat, Carolina Zorro, Ann Hickey. UK

\subsection{6/bmjpo-2021-RCPCH.12}

Background Infants requiring surgical intervention are at high risk of complications. Peri-operative checklists have demonstrated reduction in morbidity and mortality in many patient groups by maintaining high standards of care provided.

Objectives We audited compliance with current perioperative protocols on a surgical neonatal intensive care unit at King's College Hospital, London, UK and identified deficits in documentation and clinical practices. A perioperative checklist was then formulated as a quality improvement activity to standardize care which was re-audited to demonstrate significant improvisation of deficiencies.

Various new variables were introduced with the implementation of peri-operative check list to provide an extended and comprehensive health care package to infants undergoing surgical interventions. These included post-operative examination, IV antibiotics, replacement of ongoing fluid losses, identity bands and COVID swabs results prior to theatres.

Methods Review of case notes were undertaken pre and postoperatively. Documentation of clinical examination, laboratory investigations, imaging, ventilation plan, consent, communication, prescriptions of fluids, antibiotics and analgesia were assessed.

Results The case records of 48 infants (birth weight 460 4600 grams) with gestational ages ranging from 23-41 weeks were analysed (shown in table 1) both pre and post introduction of peri-operative checklist (shown in figure 1). The surgical interventions included laparotomy (32), neurosurgery (10), thoracotomy (4) and central venous line insertion (2). 